\title{
Bovine whey protein concentrate supplementation modulates maturation of immune system in suckling rats
}

\author{
Francisco J. Pérez-Cano ${ }^{1}{ }^{\dagger} \uparrow$, Silvia Marín-Gallén ${ }^{1} \dagger$, Margarida Castell ${ }^{1}$, María Rodríguez-Palmero ${ }^{2}$, \\ Montserrat Rivero ${ }^{2}$, Àngels Franch ${ }^{1}$ and Cristina Castellote ${ }^{1}$ \\ ${ }^{1}$ Department of Physiology, Faculty of Pharmacy, University of Barcelona, Spain \\ ${ }^{2}$ Laboratorios Ordesa, Barcelona, Spain
}

During neonatal life, challenges from breast milk and microbial flora promote immune system maturation. Immunonutrition in these stages may become an important way to increase natural defence systems. The aim of this study was to determine the effect of a daily bovine milk whey protein concentrate (WPC) supplement on the intestinal and systemic immune systems in suckling rats. The composition of intraepithelial and lamina propria lymphocytes (IEL and LPL) was analysed by flow cytometry. Systemic and intestinal humoral immune responses were determined by sera Ig levels and Ig-secreting cell quantification by ELISA and ELISPOT, respectively. From birth, suckling Wistar rats were supplemented with WPC or standard infant formula (SIF). The WPC group showed the same proportion of most of the main mucosal cell subsets as the reference animals. However, in the first days of life WPC enhanced the innate immunity by increasing the NK cell proportion in both epithelial and lamina propria (LP) compartments. A rise in intestinal CD8 $\alpha \alpha+$ IEL was also induced by WPC supplementation. A timecourse of sera Ig levels and spontaneous IgA, IgM and IgG production by LPL and mononuclear cells from blood and spleen, in the WPC group, exhibited a similar pattern to those pups fed only by dam's milk. In summary, the present results show the effects of WPC on enhancing mucosal innate immunity during early life.

Suckling: Rat: Intraepithelial lymphocytes: Lamina propria lymphocytes: Whey: Milk

Structural and cellular components of the immune system are present at term delivery; however, there is evidence to suggest that they are developmentally immature and may not respond appropriately to environmental stimuli ${ }^{1}$. It is during early life that important changes occur and the immune system matures, not only in systemic lymphoid organs, but also at mucosal sites. The GALT (gut-associated lymphoid tissue) is the largest immunologic organ in the body and is comprised of Peyer's patches, isolated follicles, mesenteric lymph nodes and intraepithelial and lamina propria lymphocytes (IEL and LPL) ${ }^{2}$. These intestinal immune cells are responsible for controlling the high antigenic pressure of dietary proteins and bacteria. Moreover, GALT develops during suckling, similarly to the systemic immune system, and for example, in weaning rats this particular immune compartment is not fully developed ${ }^{3,4}$. Currently, attention is focused on immunonutrition by identifying nutrients which are not only of nutritional value but also are capable of modulating immune status 5 . Undoubtedly, breast milk is the most important and beneficial food during early infancy ${ }^{6}$.

Over the last years, significant progress has been made in the characterisation of milk components affecting growth, development and functions of the gastrointestinal tract and the immune system ${ }^{7}$. In this sense, breast milk is rich in, among other things, immunoglobulins (Ig) that can bind and neutralise pathogens in the intestinal tract; bactericidal factors such as lactoferrin, lactoperoxidase and lysozyme and growth factors, nucleotides and cytokines that improve immune defence and gut-barrier function ${ }^{8}$. Colostrum is the milk produced by mammals during the first days after birth, and it is richer in these bioactive molecules than mature milk ${ }^{9}$. All these components are essential for the physiological maturation of the newborn, stimulating their immune system and providing passive protection, especially in the gastrointestinal tract, during the first days of life, the most critical period for the neonate ${ }^{10}$.

The aim of this study was to determine the effect of daily supplementation with a bovine whey protein concentrate (WPC) on the developing immune system of suckling rats by evaluating the intestinal lymphocyte phenotype and the humoral immune response.

\section{Material and methods}

Animals

Pregnant Wistar (G7) rats were obtained from Harlan (Barcelona, Spain). They were housed in individual cages under controlled temperature and humidity conditions in a

Abbreviations: GALT, Gut-associated lymphoid tissue; IE, intraepithelial; IEL, intraepithelial lymphocyte; LP, lamina propia; LPL, lamina propia lymphocyte; NK, natural killer; SIF, standard infant formula; SC, secreting cell; WPC, bovine whey protein concentrate.

* Corresponding author: Dr. Francisco J. Pérez-Cano, fax +34934035901, email franciscoperez@ub.edu

$\dagger$ Both authors contributed equally to this work 
$12 \mathrm{~h}: 12 \mathrm{~h}$ light:dark cycle, and were fed with a commercial diet (AIN-93G, Harlan) and water ad libitum. Animals were monitored daily and allowed to deliver naturally. The day of birth was identified as day 1 of life. Litters were unified to ten pups per mother, with free access to the nipples and rat diet. Studies were performed in accordance with the institutional guidelines for the care and use of laboratory animals established by the Ethical Committee for Animal Experimentation at the University of Barcelona and Catalonia Government (CEEA-UB Ref. 849/00).

\section{Dietary supplementation}

Animals were distributed in 3 groups depending on the supplementation diet: the experimental group receiving WPC; the supplemented reference group, receiving standard infant formula (SIF) and the reference group (REF) (20 animals each). Animals from the WPC group were supplemented with $0.2 \mathrm{~g} / \mathrm{kg} / \mathrm{day}$ of a premium ultra grade freeze dried bovine whey extract in water. The WPC contained $<1 \%$ fat, $75-90 \%$ protein and $18-20 \%$ carbohydrate. It also contained lactoferrin $(0.92 \%)$, IgG $(35-45 \%), \operatorname{IgA}(2 \cdot 5-5 \%)$ and $\operatorname{IgM}(3-5 \%)$ as well as high proportions of active compounds, such as natural growth factors and hormones, vitamins and amino acids, among others. Litters from the SIF group (the supplemented reference group) received $3 \mathrm{~g} / \mathrm{kg} /$ day of SIF in water. Suckling rats from the REF group received the same volumes of vehicle (mineral water). Suckling rats received the supplements daily by oral gavage throughout the lactancy period (from day 1 to 21). Low capacity syringes (Hamilton Bonaduz AG, Bonaduz, Switzerland (adapted to oral gavages (27 mm long and diameter 25 or 23) (ASICO, Westment, IL, USA( were used for oral administration before and after day 5, respectively. Litters were separated from their dams $1 \mathrm{~h}$ before oral supplementation, to allow gastric emptying in the pups. During this hour animals were identified and weighed. All daily handling was done at approximately the same time of day to avoid modifications in biological rhythms. The dose of oral supplementation was according to body weight without reaching maximum volume recommended for rats $(20 \mathrm{ml} / \mathrm{kg} / \mathrm{day})$.

\section{Blood, spleen and small intestine isolation}

Pups aged 4, 7, 14 and 21 days (suckling period) were euthanised by humanitarian methods. Blood samples were collected by cardiac puncture; both the spleen and the entire small intestine were removed. The intestines from 4 day-old rats were directly cut into $5 \mathrm{~mm}$ pieces, whereas those from 7-14 day-old rats were opened lengthwise before cutting. 21-dayold rat intestines were everted, visible Peyer's patches were excised and remaining tissue was cut.

\section{Isolation of mononuclear cells from blood and spleen}

Splenocytes were prepared following mechanical spleen disruption on a stainless steel mesh as previously described ${ }^{11}$. Mononuclear blood cells were isolated by Nycoprep ${ }^{\circledR}$ gradient centrifugation (Nycomed, Oslo, Norway). Cell numbers and viability were determined after staining dead cells with ethidium bromide (Sigma Chemical Co, St. Louis, MO, USA) and live cells with acridine orange (Sigma). Spleen or blood mononuclear cells were incubated for ELISPOT assays.

\section{Isolation and purification of intraepithelial and lamina propria lymphocytes}

Intestinal IEL and LPL suspensions were obtained by chemical and enzymatic reactions from 2-4 animals depending on their postnatal age following procedures established previously in our laboratory ${ }^{3,4}$. Both IEL and LPL suspensions were later purified and cell number and viability were determined.

\section{Immunofluorescence staining and flow-cytometer analysis}

$2 \times 10^{5}$ IEL or LPL were stained by a double immunofluorescence technique. The mouse anti-rat monoclonal antibodies conjugated to fluorescein isothiocyanate or phycoerythrin used here included anti-CD3 (1-F4), anti-CD4 (OX-35), anti$\mathrm{CD} 8 \alpha$ (OX-8), anti-CD25 (OX-39), anti-CD45 (OX-1), anti-TCR $\alpha \beta$ (R73), anti-TCR $\gamma \delta$ (V65) and anti-NKR-P1A (10/78), all from BD Pharmingen (San Diego, CA, USA); anti-CD2 (OX-34), anti-CD45RA (OX-33) and anti-CD90 (OX-7) from Caltag (Burlingame, CA, USA) and anti-CD8 $\beta$ (3.41) from Serotec (Kidlington, Oxford, UK). Staining was developed following procedures described in previous studies $^{12}$, and results were analysed with an Epics XL flow cytometer (Coulter Corp., FL, Hialeah, USA).

\section{ELISPOT and ELISA techniques}

A solid-phase enzyme-linked immunospot technique (ELISPOT) was used to quantify IgA-, IgG- and IgM- secreting cells (SC) from blood, spleen and lamina propria (LP) following the conditions established in previous studies ${ }^{3}$. Spots were automatically counted by ELISPOT-reader system (AID Diagnostica, Strassberg, Germany) and expressed as the number of Ig-SC per $10^{6}$ cells. Sera IgG, IgM and IgA concentration was determined using a sandwich ELISA technique standardised previously $^{11}$.

\section{Statistical analysis}

SPSS 10.0 (SPSS Inc, Chicago, IL, USA) was used for statistical analysis by conventional one-way ANOVA considering the experimental group as an independent variable. When supplementation had a significant effect on the dependent variable, the LSD test was applied. Significant differences were accepted when $P<0 \cdot 05$.

\section{Results}

Animal growth

Body weight increase was unaffected by either daily WPC or SIF supplementation during the first half of the suckling period, and showed growth similar to the reference group. However, during late suckling, higher body weight gain was found in those animals supplemented with $\operatorname{SIF}(P<0 \cdot 05$, data not shown). 


\section{IEL composition in small intestine}

WPC supplemented animals showed the same percentages in most of the main subsets within the intraepithelial (IE) compartment as the reference group during suckling period: total gated IE leukocytes (CD45+, 90\%), IE-T lymphocytes (CD3+, $\sim 60$ to $90 \%$ from day 4 to 21 , respectively), and CD8+ IEL ( $~ 80-50 \%$ from day 4 to 21 , respectively). Moreover, in all studied groups, the TCR $\alpha \beta+$ cell proportion in CD8+ IEL increased from $\sim 25 \%$ (day 4 ) to $\sim 50 \%$ (day 21 ), and the TCR $\gamma \delta+$ cell percentage in CD8+ IEL rose from $\sim 15 \%$ (day 4 ) to $\sim 25 \%$ (day 21 ).

Supplementation with WPC induced an increase in NKR$\mathrm{P} 1 \mathrm{~A}+$ (a rat NK cell marker) cell proportion in the IE compartment compared to both the reference and SIF groups at the beginning of the suckling period $(P<0 \cdot 05$, Fig. 1A). Later, all groups showed a similar development pattern for this subset. Moreover, the CD8+ cell proportion in NKR$\mathrm{P} 1 \mathrm{~A}+\mathrm{IEL}$ markedly decreased during the entire suckling period in all studied groups (data not shown).

The CD $8 \alpha \alpha / C D 8 \alpha \beta$ ratio in IEL, which may represent the relationship between lymphocytes developed in the intestinal environment with respect to cells with typical phenotype from extra-mucosal sites, was modified by WPC supplementation.

A

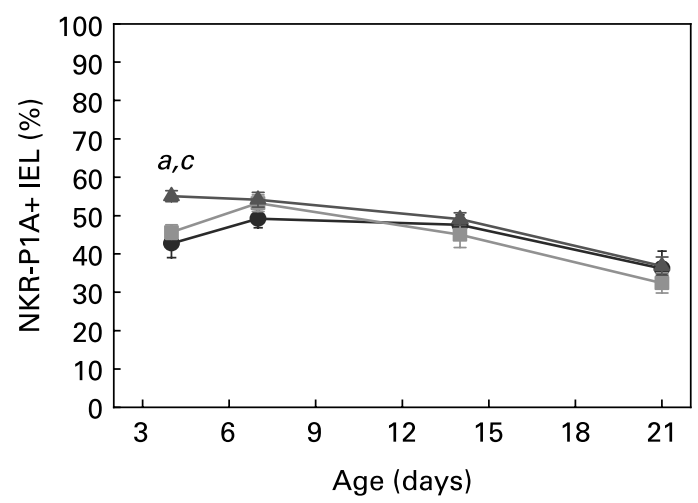

C

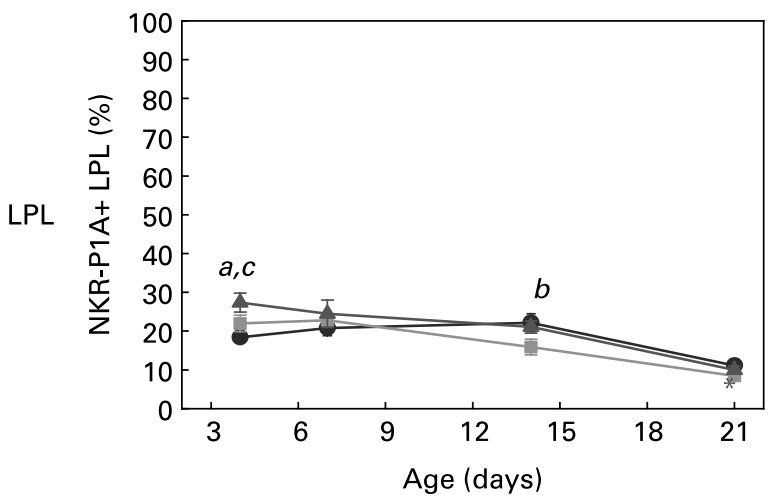

Thus, the $\operatorname{CD} 8 \alpha \alpha / C D 8 \alpha \beta$ ratio in the WPC group was higher than in the reference and SIF groups, from day 7 and throughout the entire suckling period $(P<0 \cdot 05$, Fig. 1B).

\section{LPL composition in small intestine}

Total gated leukocytes in LP $(\mathrm{CD} 45+$ ) rose from $\sim 65 \%$ (day 4$)$ to $\sim 95 \%$ (day 21) in all groups. There was a physiological increase of B cell proportion (CD45RA+ cells) during suckling in LP that was not modified by the supplements during this period. Moreover, the proportion of CD90 molecules in B cell surfaces, a negative marker of maturation, was $\sim 75 \%$ in the three experimental groups during suckling (data not shown).

The percentage of CD4+ LPL ranged between $\sim 10-30 \%$ during suckling in all three groups, and the proportion of these cells bearing CD3 (typical T cell marker), CD2 (adhesion molecule), CD25 ( $\mathrm{T}$ cell activation marker) and CD90, were not modified by the supplements. With respect to CD8+LPL, $\mathrm{CD} 8 \alpha \alpha / \mathrm{CD} 8 \alpha \beta$ ratio and $\mathrm{TCR} \alpha \beta$ percentages were similar among WPC, SIF and reference groups (data not shown).

At day 4 of life, similar to the IE compartment, the NKR$\mathrm{P} 1 \mathrm{~A}+$ cell proportion in LPL in WPC supplemented animals $(\sim 27 \%)$ was higher than those in the SIF $(\sim 21 \%)$ and reference $(\sim 17 \%)$ groups $(P<0 \cdot 05$, Fig. 1C). However, at the end

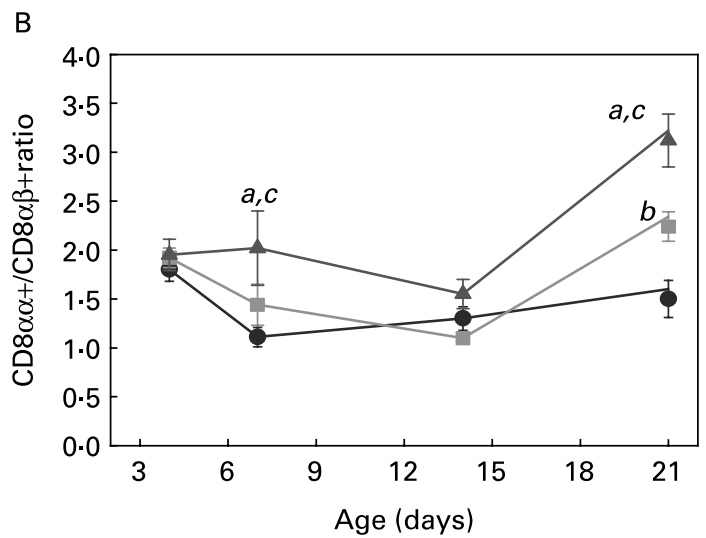

D

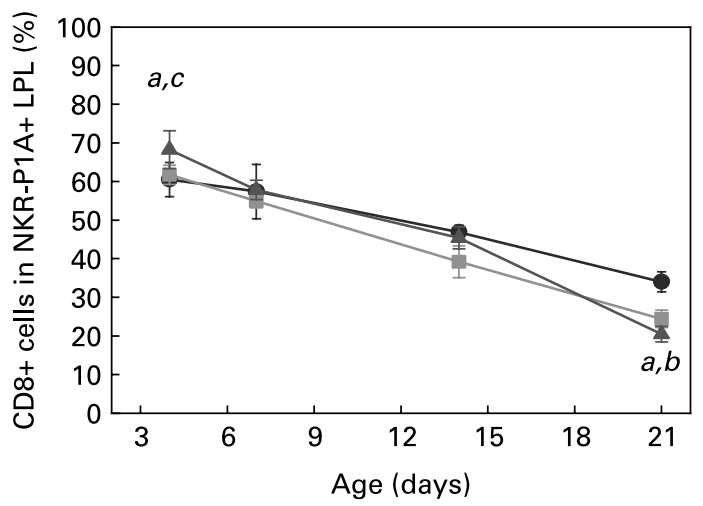

Fig. 1. Developmental time-course of intestinal cells during the suckling period under supplementation conditions. (A) NKR-P1A + cells with respect to total IEL, (B) $C D 8 \alpha \alpha+/ C D 8 \alpha \beta+$ ratio in total IEL, (C) NKR-P1A + cells with respect to total LPL, and (D) NKR-P1A + CD8 + LPL with respect to NKR-P1A + LPL. Each data corresponds to the mean $\pm \operatorname{SEM}(N=5-10$ animals). Statistical differences: $a P<0.05$ WPC vs. Reference, $b P<0.05$ SIF vs. Reference, $c P<0.05$ WPC vs. SIF. 
of the suckling period the NKR-P1A+ LPL proportion in the WPC group did not differ from that of the other groups. Moreover, the youngest (day 4) WPC supplemented animals showed a higher proportion of NKR-P1A+ LPL bearing $\mathrm{CD} 8+$ marker compared to the reference and SIF groups $(P<0 \cdot 05$, Fig. 1D). On the other hand, at the end of the suckling period a decrease in this subset in the WPC and SIF groups was observed $(P<0 \cdot 05$, Fig. 1D).

\section{Spontaneous Ig production by blood, spleen and LP mononuclear cells}

Since B cells represent a predominant subset during suck$\operatorname{ling}^{3,11}$, we studied the ability to spontaneously secrete $\mathrm{IgG}$, $\operatorname{IgM}$ and IgA antibodies by means of ELISPOT in different immune compartments. LP cells showed no IgA-SC or IgGSC throughout suckling period. On day 21, the number of IgM-SC was similar in all groups $\left(50-115 \operatorname{IgM}-\mathrm{SC} / 10^{6}\right.$ cells, Table 1). Spleen neonatal cells barely secreted $\mathrm{IgG}$ or IgA but did produce IgM. The number of IgM-SC increased in the spleen after the first week of life similar to the WPC and reference groups: $\left(\sim 300 \mathrm{IgM}-\mathrm{SC} / 10^{6}\right.$ cells during first week of life and $\sim 1000 \mathrm{IgM}-\mathrm{SC} / 10^{6}$ cells at the end of suckling. Blood Ig-SC numbers were very low for all Ig isotypes in suckling rats $\left(\left(40 \mathrm{Ig}-\mathrm{SC} / 10^{6}\right.\right.$ cells, Table 1$)$. Effects of WPC on antibody secretion were not found.

\section{Serum Ig levels}

Serum IgG and IgM levels were detected from the first week and increased until day 21 with values ranging between $\sim 0.5-2.5 \mathrm{mg} / \mathrm{ml}$ and $\sim 5-150 \mu \mathrm{g} / \mathrm{ml}$, respectively. $\operatorname{IgA}$ levels rose more than 100 times only during last week of suckling $(\sim 75-1000 \mathrm{ng} / \mathrm{ml})$. Any serum Ig time-course was not substantially modified by supplementation with WPC in comparison to the reference group. Data from day 21 are shown in Table 1 as a summary of these results.

\section{Discussion}

A vast body of literature is focused on the associated effects of bioactive compounds present in milk and colostrum on immune health. However, little is known about the influence of these dairy products on the mucosal and systemic immune system maturation during suckling.

In this study, most of the main intestinal lymphocyte subsets, i.e. CD45+ (total leukocytes) (CD3+, TCR $\alpha \beta+$, $\mathrm{TCR} \gamma \delta+$ and CD4+ (T cells) and CD45RA+ (B cells), followed the same time-course in WPC supplemented suckling rats as they did in the reference group. However, WPC supplementation from birth increased NKR-P1A ${ }^{+}$cell proportion in early life (day 4 ) in both intestinal compartments consider Ed. NK cells constitute the first line of GALT defence and, as reported previously, they represent a high proportion of rat IE and LP compartments during the first days of life, decreasing with age $\mathrm{e}^{3,4,13}$. Our results showed that bioactive compounds present in WPC, by increasing NK cell proportion in GALT, enhanced innate immunity in early life. A similar effect in spleen from adult mice fed with colostrum has been described ${ }^{14}$. An increase in the NK cell proportion after lactoferrin administration has been found in circulating cells in adult rats ${ }^{15}$ and in intestinal cells in mice ${ }^{16}$. Moreover, our data derived from the end of suckling (day 21) suggested that NKR-P1A+ LPL from WPC supplemented animals exhibited a more mature intestinal phenotype (NKR-P1A+ CD8-), which corresponds to that found in adult age, than reference animals did.

Intestinal CD8 $\alpha \alpha+$ IEL correspond to a non-conventionally selected lymphocyte population that develops in the gut microenvironment and expresses this particular isoform of CD8 co-receptor ${ }^{17}$. Our results showed an increase throughout the entire suckling period in the $\mathrm{CD} 8 \alpha \alpha+/ \mathrm{CD} 8 \alpha \beta+$ IEL ratio in the WPC group. These data suggest that WPC enhances immune responses developed specifically at mucosal sites by increasing the proportion of gut CD8 $\alpha \alpha+$ IEL, perhaps by means of a local effect of WPC compounds. Similar to an NK cell increase derived from lactoferrin consumption, a rise in $\mathrm{CD} 8+$ intestinal cells was also found elsewhere ${ }^{16}$.

The present study shows that WPC promotes the expansion during early age of cell subsets involved in innate and mucosal immune response. This is another mechanism by which they may contribute to the largely studied properties and benefits of colostral and whey proteins in preventing gastrointestinal infections ${ }^{14-18}$. This effect may be partially attributed to the high concentration of lactoferrin present in the dairy product used in the present study $(9.2 \mathrm{mg} / \mathrm{g}$ of dried WPC).

The immunoenhancing effect of whey proteins on the production of antibodies against a specific antigen has been well documented ${ }^{19}$. Our results showed that WPC supplementation failed to enhance the humoral immune response during suckling (blood, LP and spleen) in the absence of specific stimulation. This result could be explained by the low ability of immune cells to produce Ig during this period ${ }^{3,11}$. However, the dairy supplementation used in this study did not disturb the physiological acquisition of Ig production capacity, as

Table 1. Effect of WPC and SIF supplementation on humoral immune response in suckling rats

\begin{tabular}{|c|c|c|c|c|c|c|c|c|c|c|c|c|}
\hline \multirow[b]{3}{*}{ Group } & \multicolumn{6}{|c|}{ Serum Ig isotype concentrations* } & \multicolumn{6}{|c|}{$\operatorname{lgM}-\mathrm{SC}\left(/ 10^{6}\right.$ cells $)$} \\
\hline & \multicolumn{2}{|c|}{$\lg G(\mu \mathrm{g} / \mathrm{ml})$} & \multicolumn{2}{|c|}{$\operatorname{lgM}(\mu \mathrm{g} / \mathrm{ml})$} & \multicolumn{2}{|c|}{$\operatorname{lgA}(\mathrm{ng} / \mathrm{ml})$} & \multicolumn{2}{|c|}{ Spleen* } & \multicolumn{2}{|c|}{ Blood $^{*}$} & \multicolumn{2}{|c|}{ LP† } \\
\hline & Mean & SEM & Mean & SEM & Mean & SEM & Mean & SEM & Mean & SEM & Mean & SEM \\
\hline WPC & 2466.0 & 111.5 & 129.5 & 18.9 & $1001 \cdot 1$ & 108.9 & $856 \cdot 7$ & 353.3 & $4 \cdot 10$ & 2.3 & 106.0 & 23.5 \\
\hline SIF & $2146 \cdot 3$ & 335.2 & $127 \cdot 4$ & 23.6 & 734.8 & 71.7 & $1060 \cdot 9$ & 141.9 & $4 \cdot 10$ & $2 \cdot 6$ & 52.4 & $27 \cdot 3$ \\
\hline Reference & 2392.3 & 110.4 & 147.6 & $7 \cdot 2$ & $1012 \cdot 6$ & 139.7 & 935.5 & $222 \cdot 4$ & 4.88 & 2.5 & 114.5 & $28 \cdot 3$ \\
\hline
\end{tabular}

Results correspond to $14(\dagger)$ or $21\left(^{*}\right)$ day-old animals. Number of IgM-secreting cells (SC) are expressed with respect to $10^{6}$ cells. $N=5-10$ animals per experimental group. 
evidenced by the similar pattern of development found in all of the experimental groups.

In summary, this study demonstrates that daily supplementation with WPC during suckling improves the development of intestinal and innate cells: CD8 $\alpha \alpha+$ IEL and NK cells in IE and LP compartments.

\section{Conflict of interest statement}

The present study was supported by grants from the Spanish Health and Consumption Ministry (CIBER 06/02/0079), from the Generalitat de Catalunya (SGCR-2005-00 833) and from Laboratorios Ordesa, S.L. (FBG-301671). SMG was holder of a fellowship from The University of Barcelona. FJPC and SMG contributed equally to this work.

\section{Acknowledgements}

We also thank the "Serveis Científico-Tècnics" of the University of Barcelona, especially Dr. J. Comas, for expert assistance in flow cytometry.

\section{References}

1. Walker WA (2002) Development of the intestinal mucosal barrier. J Pediatr Gastroenterol Nutr 34, S33-S39.

2. Mowat AM (2003) Anatomical basis of tolerance and immunity to intestinal antigens. Nat Rev Immunol 3, 331-341.

3. Pérez-Cano FJ, Castellote C, Marín-Gallén S, Franch A \& Castell M (2005) Neonatal immunoglobulin secretion and lymphocyte phenotype in rat small intestine lamina propria. Pediatr Res 58, 164-169.

4. Pérez-Cano FJ, Castellote C, González-Castro A, Pelegrí C, Castell M \& Franch A (2005) Developmental changes in intraepithelial T lymphocytes and NK cells in the small intestine of neonatal rats. Pediatr Res 58, 885-891.

5. DeWitt RC \& Kudsk KA (1999) The gut's role in metabolism, mucosal barrier function, and gut immunology. Infect Dis Clin North Am 13, 465-481.

6. Lönnerdal B (2000) Breast milk: a truly functional food. Nutrition 16, 509-511.
7. Hanson LA, Korotkova M \& Telemo E (2005) Human milk: its components and their immubiologic functions. In Mucosal Immunology, 3rd ed., vol.1, pp.1795-1827 [J Mestecky, J Bienenstock, M Lamm, W Strober, J McGhee and LI Mayer, editors]. Amsterdam: Elsevier Academic Press.

8. Gill HS, Doull F, Rutherfurd KJ \& Cross ML (2000) Immunoregulatory peptides in bovine milk. Br J Nutr 84, S111-S117.

9. Uruakpa FO, Ismond MAH \& Akobondu ENT (2002) Colostrum and its benefits: a review. Nutr Res 22, 755-767.

10. Kelleher SL \& Lonnerdal B (2001) Immunological activities associated with milk. Adv Nutr Res 10, 39-65.

11. Pérez-Cano FJ, González-Castro AM, Marín-Gallén S, Castellote C, Franch A \& Castell M (2007) Phenotypic and functional characteristics of rat spleen lymphocytes during suckling. Dev Comp Immunol 9, [Epub ahead of print].

12. Ramiro-Puig E, Pérez-Cano FJ, Ramírez-Santana C, Castellote C, Izquierdo-Pulido M, Permanyer J, Franch A \& Castell M (2007) Spleen lymphocyte function modulated by cocoa-enriched diet. Clin Exp Immunol 12, [Epub ahead of print].

13. Todd DJ, Greiner DL, Rossini AA, Mordes JP \& Bortell R (1999) An atypical population of NK cells that spontaneously secrete IFN- $\gamma$ and IL- 4 is present in the intraepithelial lymphoid compartment of the rat. J Immunol 167, 3600-3609.

14. Xu LB, Chen L, Gao W \& Du KH (2006) Bovine immune colostrum against 17 strains of diarrhea bacteria and in vitro and in vivo effects of its specific IgG. Vaccine 24, 2131-2140.

15. Sekine K, Ushida Y, Kuhara T, et al. (1997) Inhibition of initiation and early stage development of aberrant crypt foci and enhanced natural killer activity in male rats administered bovine lactoferrin concomitantly with azoxymethane. Cancer Lett 121, 211-216.

16. Wang WP, Iigo M, Sato J, Sekine K, Adachi I \& Tsuda H (2000) Activation of intestinal mucosal immunity in tumorbearing mice by lactoferrin. Jpn J Cancer Res 91, 1022-1027.

17. Hayday A, Theodoridis E, Ramsburg E \& Shires J (2001) Intraepithelial lymphocytes: exploring the Third Way in immunology. Nat Immunol 2, 997-1003.

18. Florén CH, Chinenye S, Elfstrand L, Hagman C \& Ihse I (2006) ColoPlus, a new product based on bovine colostrum, alleviates HIV-associated diarrhoea. Scand J Gastroenterol 41, 682-686.

19. Wong CW \& Watson DL (1995) Immunomodulatory effects of dietary whey proteins in mice. J Dairy Res 62, 359-368. 